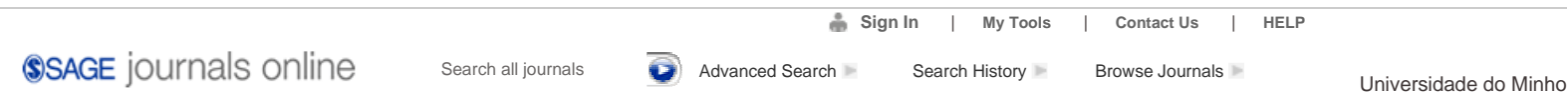

\title{
Studies on Preform Properties of Multilayer Interlocked Woven Structures Using Fabric Geometrical Factors
}

Naveen V. Padaki

Regional Silk Technological Research Station, CSTRI, Central Silk Board, Guwahati - 781022, India

R. Alagirusamy

Department of Textile Technology, Indian Institute of Technology-Delhi, Hauz Khas, New Delhi - 110 016, India, alagiru@gmail.com

B.L. Deopura

Department of Textile Technology, Indian Institute of Technology-Delhi, Hauz Khas, New Delhi - 110 016, India

R. Fangueiro

Department of Textile Engineering, University of Minho, 4800-058-Guimarães, Portugal

Abstract

Structure property correlation is a critical textile research area explored by various researchers and many factors have been proposed over the years to predict/compare/design the woven fabrics. Cross-over firmness factor (CFF) and floating yarn factor (FYF) have been recently proposed as parameters to understand weave effect on fabric properties (Morino, H., Matsudaira, M. and Furutani, M. (2005). Predicting Mechanical Properties and Hand Values from the Parameters of Weave Structures, Textile Research Journal, 75(3): 252-257). Redefined CFF and FYF factors using fabric fields in terms of interlacement index $(\mathrm{I})$ and float index $(\mathrm{F})$, respectively have been proposed in this article. This new approach provides better understanding of the interlacements and floats in the woven structure and further they are applied on multilayer interlocked fabrics to quantify the structural influence on the properties. Multilayer interlocked woven fabrics with different interlacement patterns have been developed. Influence of fabric structure on preform properties relevant for resin transfer molding composite manufacture, such as compression, permeability, and tensile behavior were studied with respect to the interlacement and float indices. Tensile and compression tests were conducted on universal testing machine. Liquid permeability of these structures was evaluated based on horizontal wicking and contact angle wettability tests. Results show that influence of structural factor is greater on tensile and permeability properties than the compression properties of these multilayer fabrics.

multilayer interlocked structures firmness factor CFF interlacement index float index compression properties permeability 